\title{
ORGANIZAÇÃO E REPRESENTAÇÃO DE ÁREAS DO CONHECIMENTO EM CIÊNCIA E TECNOLOGIA: princípios de agregação em grandes áreas segundo diferentes contextos de produção e uso de informação ORGANIZATION AND REPRESENTATION OF KNOWLEDGE AREAS IN SCIENCE AND TECHNOLOGY: principles of aggregation in great areas according to different contexts of information production and use
}

Rosali Fernandez de Souza, PhD

Pesquisadora Titular IBICT/MCT

Professora do Programa de Pós-graduação em Ciência da Informação, convênio IBICT-UFF

rosali@,ibict.br

\begin{abstract}
Resumo
A Organização do Conhecimento é área tradicional de pesquisa e ensino em Ciência da Informação. Grande parte da literatura nesta área trata do processo, das atividades e dos instrumentos especialmente desenvolvidos no tratamento de documentos para armazenamento, disseminação, recuperação e uso em sistemas e serviços de informação. Os diferentes tipos e demandas de informação têm demonstrado a crescente diversidade em organização do conhecimento em outros contextos de aplicação. O presente trabalho é de natureza exploratória. Analisa exemplos de tabelas e esquemas de classificação em C\&T com o objetivo de identificar agregações em nível de super-ordenação de áreas do conhecimento para diferentes necessidades de produção e uso de informação. Os instrumentos analisados foram: esquemas de classificação bibliográfica, tabelas de classificação de áreas do conhecimento para propósitos de comunicação em ciência, administração de programas de agências de fomento a para a produção de estatísticas nacionais. Apresenta também uma abordagem temática de classificação dos saberes no contexto da educação. Os resultados mostraram que há consenso na agregação de áreas em grandes áreas do conhecimento em $\mathrm{C} \& \mathrm{~T}$, embora ocorram diferenças no número e na ordem de apresentação das grandes áreas em função da natureza do objeto de representação, assim como da finalidade da organização do conhecimento.
\end{abstract}

Palavras-chave: Organização do Conhecimento. Representação da Informação. Classificações em Ciência e Tecnologia. Áreas do Conhecimento.

\section{CONSIDERAÇÕES PRELIMINARES}

Tradicionalmente, as atividades de organização do conhecimento e representação da informação estiveram relacionadas a sistemas de recuperação de documentos. Os esquemas de classificação, gerais e especializados, os tesauros, entre outros tipos de instrumentos, foram criados para a organização física de acervos ou para a representação temática do conteúdo intelectual dos documentos visando acesso, disseminação e recuperação sistemática. Muitos desses instrumentos tiveram princípios norteadores oriundos da Filosofia e foram desenvolvidos segundo métodos e técnicas diferentes de abordagem do conhecimento, no intuito de aprimorar a recuperação de informação em diferentes contextos de produção e uso.

A atividade de organização e representação do conhecimento também se faz presente no âmbito da gestão e avaliação, porém, sob uma ótica peculiar diferente das necessidades das 
bibliotecas e outros sistemas de recuperação de documentos. As agências de fomento em C\&T, por exemplo, visam sistematizar a informação sobre $C \& T$ e não a informação de $C \& T$ para seus fins específicos de atuação, contexto ainda pouco explorado no âmbito da Ciência da Informação. Esta caracterização evidencia diferenças fundamentais de pontos de vista de um mesmo universo segundo princípios especialmente definidos para o tratamento da informação. Portanto, a pesquisa na área de organização e representação do conhecimento no contexto de gestão e avaliação não deixa de ser ainda um desafio para os cientistas da informação.

As Agências de Fomento em C\&T, no desempenho de suas finalidades de gestão e avaliação como suporte ao desenvolvimento de políticas públicas, lidam com as áreas do conhecimento como elemento referencial de categorização desse universo principalmente no contexto do ensino, da pesquisa e da inovação. Para tal, a necessidade de classificação está diretamente relacionada à praticidade na sistematização de C\&T para gerenciar e avaliar os programas das agências.

No presente trabalho consideramos importante refletir sobre a organização e representação do conhecimento em diferentes contextos de uso de informação em C\&T. O trabalho é de natureza exploratória. Tem como objetivo identificar princípios norteadores de agregação de áreas do conhecimento no nível de super-ordenação em grandes áreas em tabelas e esquemas de classificação elaborados para propósitos diferentes.

A diversidade de abordagem selecionada contempla classificações para organização de acervos bibliográficos em bibliotecas, classificação de comunicações em eventos científicos, classificação de projetos de pesquisa e desenvolvimento para financiamento e classificação temática no âmbito do ensino educativo dos saberes.

\section{ESTRUTURAS DE CLASSIFICAÇÃO EM CIÊNCIA E TECNOLOGIA DEFINIDAS PARA DIFERENTES CONTEXTOS DE PRODUÇÃO E USO DE INFOMAÇÃO}

A Organização do Conhecimento, pela sua importância em sistemas de recuperação de informação, sempre mereceu papel de destaque no ensino e na pesquisa na área da Ciência da Informação. A revista especializada Knowledge Organization (1993) indica em seu subtítulo como pontos centrais de referência dessa área a Teoria do Conceito, a Classificação, a Indexação e a Representação do Conhecimento. 
Langridge (1977) considera a classificação como a principal atividade na organização do conhecimento. Ressalta que tabelas, esquemas de classificação e demais linguagens de representação são construídas frente a propósitos definidos. Langridge destaca também o importante papel da classificação na educação, papel que só mais recentemente tem sido evidenciado.

O processo de classificar é um processo meio que se desenvolve em base de dois referenciais básicos: a natureza da informação objeto de classificação e as caraterísticas e necessidades específicas da comunidade usuária frente ao propósito último de uso da informação, o que constitui, em síntese, o leitmotif da construção das linguagens de representação.

A área da Ciência da Informação possui vasta literatura sobre questões relacionadas à classificação e indexação da produção científica publicada em áreas de C\&T. As décadas de 50, 60 e 70 tiveram uma produção particularmente representativa na Inglaterra. Uma série clássica que merece destaque é aquela composta por três livros básicos sobre classificação e indexação: nas Ciências (VICKERY, 1958), nas Ciências Sociais (FOSKETT, 1963) e nas Humanidades (LANGRIDGE, 1976). Importante ressaltar que a abordagem do universo de C\&T em três grandes áreas denominadas como Ciências, Ciências Sociais e Humanidades indicava particularidades inerentes a cada uma delas e já evidenciava uma macro estrutura de organização de áreas do conhecimento em grandes áreas.

Sayer's e Palmer, autores clássicos da área de classificação, atentam para um ponto crucial da presente discussão. Ambos, sob abordagens diferentes, evidenciam que toda classificação está relacionada a um determinado propósito e, como tal, este propósito tem que ser claramente definido antes da construção, adaptação ou adoção de uma classificação em qualquer contexto de aplicação visando a recuperação de informação.

SAYER'S (1967) numa abordagem histórica da classificação em bibliotecas evidencia que diferentes pontos de vista de uso de informação foram identificados no estabelecimento de princípios de classificação na representação do conhecimento em tabelas e esquemas de classificação, principalmente bibliográficos. Mostra que, embora de naturezas muito diferentes, os esquemas produzidos foram igualmente válidos em determinadas épocas, culturas e contextos de uso, sempre mantidas as circunstâncias de origem e os fins específicos para os quais foram criados.

PALMER (1971) aborda o papel da classificação no contexto da Educação. É importante destacar os elementos que selecionou para sua abordagem no âmbito das bibliotecas: o embasamento sócio-histórico da classificação, a formação das classes principais 
e seu questionamento contemporâneo, a contribuição de Ranganathan e, a classificação como estudo fundamental para o profissional bibliotecário. Evidencia a inquestionável importância de cada um destes elementos no estudo da classificação, o que pode certamente ser extrapolado para outras finalidades de classificação além da biblioteca.

Estudos mais modernos como o de ANDERSEN (2002) apontam para a importância

de se agregar enfoques históricos, educacionais, culturais e sociais do conhecimento às questões tradicionais da área de organização e representação no contexto da recuperação de informação. Estes aspectos são importantes uma vez que a classificação deve acompanhar a dinâmica de desenvolvimento do conhecimento.

As classificações selecionadas para análise são de naturezas diferentes visando ilustrar aspectos da organização e representação do conhecimento em diferentes abordagens do universo de C\&T. Assim, foram escolhidos: esquemas gerais de classificação bibliográfica, tabelas de classificação de comunicações apresentadas em eventos científicos, classificações para projetos de pesquisa e desenvolvimento em uso no país e no exterior e classificação de saberes científicos no contexto da educação. A seguir, a identificação e análise das fontes selecionadas.

\section{a) Esquemas de classificação bibliográfica}

Como sabemos, os esquemas de classificação bibliográfica foram construídos em base de abordagens de cunho filosófico para a sistematização do universo de conhecimento. No intuito de identificar diferentes princípios que nortearam as estruturas desses esquemas na agregação das áreas do conhecimento em classes principais, selecionamos os exemplos que consideramos mais representativos quanto à ordenação adotada na representação das grandes áreas do conhecimento.

Entre os esquemas gerais de classificação bibliográfica existentes, escolhemos para análise: a Classificação Decimal de Dewey, a Classificação de Assunto de Brown, a Classificação Bibliográfica de Bliss, a Classificação de Dois Pontos de Ranganathan e a Classificação da Biblioteca do Congresso (USA).

A Classificação Decimal de Dewey apresenta uma sistematização do universo do conhecimento em dez classes principais, que se mantiveram praticamente inalteráveis ao longo do tempo desde a publicação da primeira edição em 1876. A base do esquema foi influenciada por Harris que por sua vez foi influenciado por Bacon, estabelecendo Memória, Imaginação e Razão como seqüência lógica das nove classes principais que correspondem a grosso modo, às disciplinas fundamentais do conhecimento. A Razão representada por 
Filosofia, Religião, Ciências Sociais, Filologia, Ciências Puras e Ciências Aplicadas (Tecnologia); a Imaginação representada por Artes e Literatura; a Memória representada pela História, Geografia e Biografia. O esquema compreende também uma classe principal 000 Obras Gerais que, como sua denominação indica, agrega áreas que seu idealizador identificou como de caráter geral, tendo relação com todo o esquema. A mesma base do esquema de Dewey foi adotada pela Classificação Decimal Universal.

A base do sistema da Classificação de Assunto de Brown, cuja primeira edição data de 1906, segue a ordem do aparecimento das coisas, no tempo: a Matéria e a Força geram a Vida, esta produz Inteligência e a Inteligência, o Registro dos Fatos.

Assim, a divisão dos conhecimentos humanos compreende quatro grandes grupos: Matéria e Força representando as Ciências Físicas; Vida representada pelas Ciências Biológicas, Etnologia e Medicina, Biologia Econômica (incluindo Agricultura, Medicina Veterinária) e Artes Domésticas; Inteligência representada por Filosofia e Religião, Ciências Sociais e Políticas; Registro representando Língua e Literatura, Formas Literárias, História e Geografia e a Biografia.

Podemos dizer que a seqüência de ordenação do esquema de classificação de Brown está na mesma linha de raciocínio filosófico que embasou o esquema de Dewey. Inicia pelas ciências físicas e biológicas, seguidas das ciências humanas e sociais, terminando nos registros do conhecimento nas diferentes formas de expressões humanas.

A Classificação Bibliográfica de Bliss apresenta uma estrutura segundo o "consenso científico e educacional', seguindo uma ordem de implicação da idéia de evolução. O esquema de Bliss adotou a divisão dos conhecimentos humanos conforme o conceito educacional da época de sua construção (1940), reunindo-os em quatro grandes grupos: Filosofia, Ciência, História, Tecnologia e Arte.

O esquema de Bliss inicia pela Filosofia, revelando a sua importância no contexto científico-educacional, seguida da Ciência. Destaca a História como classe principal e termina a seqüência com Tecnologia e Arte. Observa-se que Filosofia, História e Arte são consideradas grandes áreas, no mesmo nível de Ciência e Tecnologia.

A Classificação de Dois Pontos de Ranganathan, cuja primeira edição data de 1933, apresenta cerca de quarenta classes principais ordenadas na seqüência: Ciências e Tecnologia, Misticismo, Artes, Humanidades e Ciências Sociais.

Observamos que Ranganathan inicia a ordenação das classes principais por Ciências e Tecnologia representando as áreas do conhecimento de cunho teórico e aplicado. Em 
conseqüência de sua formação religiosa, destaca o Misticismo. Segue com as Artes e depois com as Humanidades e as Ciências Sociais.

A Classificação da Biblioteca do Congresso (EUA), apresenta vinte classes principais ordenadas na seqüência Humanidades, Ciências Sociais, Artes, Ciência e Tecnologia.

A seqüência de ordenação da Biblioteca do Congresso iniciada pelas Humanidades, seguida das Ciências Sociais, reflete naturalmente a sua origem de especialidade em legislação e áreas afins. Quando a Biblioteca do Congresso incorporou a coleção pessoal doada pelo presidente Thomas Jefferson após incêndio, expandiu seu acervo para artes e ciência e tecnologia.

Em conjunto, os princípios de divisão das grandes áreas adotados pelos esquemas de classificação bibliográfica, analisados revelam embasamento de origem filosófica e características próprias da cultura dos classificacionistas.

Como pode ser observado no QUADRO 1, o conjunto desses dados revela uma coincidência de denominação nas agregações de super-ordenação das grandes áreas e classes principais. No entanto, há diversidade, chegando mesmo à inversão, na seqüência de ordenação das grandes áreas nos diferentes esquemas analisados.

\section{Quadro 1 Princípios de Divisão e Grandes Áreas do Conhecimento em Esquemas de}

\begin{tabular}{|l|l|l|l|l|}
\hline $\begin{array}{c}\text { Classificação } \\
\text { Decimal de Dewey e } \\
\text { Classificação } \\
\text { Decimal Universal }\end{array}$ & $\begin{array}{c}\text { Classificação de } \\
\text { Assunto de Brown }\end{array}$ & $\begin{array}{c}\text { Classificação } \\
\text { Bibliográfica de } \\
\text { Bliss }\end{array}$ & $\begin{array}{c}\text { Classificação da } \\
\text { Biblioteca do } \\
\text { Congresso dos } \\
\text { EEUU }\end{array}$ & $\begin{array}{c}\text { Classificação de } \\
\text { Dois Pontos de } \\
\text { Ranganathan }\end{array}$ \\
\hline Razão & Matéria e Força & Filosofia & Humanidades & Ciências e Tecnologia \\
\hline Filosofia & Ciências Físicas & Ciência & Ciências Sociais & Misticismo \\
\hline Religião & Vida & História & Artes & Artes \\
\hline Ciências Sociais & Ciências Biológicas & Tecnologia e Arte & Ciência e Tecnologia & Humanidades \\
\hline Filologia & Etnologia & & & \\
\hline Ciências Puras & Medicina & & & Ciências Sociais \\
\hline Ciências Aplicadas & Biologia & & & \\
\hline Imaginação & Inteligência & & & \\
\hline Artes & Filosofia e Religião & & & \\
\hline Literatura & Ciência Social e Política & & & \\
\hline Memória & Registro & & & \\
\hline História & Língua e Literatura & & & \\
\hline Geografia & Formas Literárias & & & \\
\hline Biografia & História e Geografia & & & \\
\hline & Biografia & & & \\
\hline & & & & \\
\hline
\end{tabular}




\section{b) Seções de classificação das comunicações apresentadas às Reuniões Anuais da Sociedade Brasileira para o Progresso da Ciência (SBPC) no tempo}

Um exemplo interessante de ser analisado, no escopo do presente trabalho, é o da SBPC na classificação das comunicações apresentadas às reuniões anuais quanto ao número e à ordenação de áreas do conhecimento em grandes áreas no tempo: de 1956 a 2005.

Até 1975, havia uma enumeração de vinte e cinco áreas do conhecimento, sem agregação hierárquica em grandes áreas. No período de 1976 a 2005, ocorreu o agrupamento das áreas em diferentes estruturas, correspondendo ao mínimo três grandes áreas no ano de 2001 e ao máximo de oito em 2005. Podemos estabelecer como principais marcos de mudança na estrutura da classificação quanto à ordenação das áreas e grandes áreas no tempo, os anos de 1976, 1984, 2001 e 2003.

Ainda sem uma estrutura de grandes áreas até 1976, a ordem das áreas começava pelas Ciências Matemáticas, da Matéria, da Terra e do Universo, continuava pelas Ciências da Vida e terminava com as Ciências Humanas e Sociais. Em 1976, ano que corresponde à primeira estrutura nítida das áreas em grandes áreas, a seqüência anterior das grandes áreas é acrescida de uma nova grande área, Ciências Aplicadas, que permanece até 1984.

Em 1984 a seqüência das grandes áreas passa a ser alfabética, modificando a ordenação da estrutura da classificação até então estabelecida para: Ciências Aplicadas, Ciências do Homem, Ciências Matemáticas, Ciências da Matéria, Ciências do Meio Ambiente, Ciências da Terra e do Universo e Ciências da Vida. Em 2001 o número de grandes áreas é de três com a seguinte seqüência de ordenação: Ciências Humanas; Ciências Biológicas e da Saúde; e Ciências Exatas e Tecnológicas. Em 2003 o número de grandes áreas passa a ser de oito na seguinte ordenação: Ciências Exatas e da Terra; Engenharias; Ciências Biológicas; Ciências da Saúde; Ciências Agrárias; Ciências Sociais Aplicadas; Ciências Humanas; Artes, Letras e Lingüística - ordem inversa do período anterior, seguindo a tabela em vigor do CNPq.

$\mathrm{Na}$ análise das tabelas de classificação da SBPC foi observado que as grandes áreas Ciências Aplicadas e Ciências do Meio Ambiente apareceram e desapareceram como tal no tempo.

A grande área Ciências Aplicadas aparece como grande área no período 1976-2000, compreendendo áreas oriundas das Ciências Exatas e Tecnológicas, das Ciências Biológicas e das Ciências Humanas e Sociais.

A grande área Ciências do Meio Ambiente aparece apenas no período de 1976 a 1994. $\mathrm{Na}$ ordenação das seções aparece intercalada na seqüência das grandes áreas das Ciências 
Exatas e Tecnológicas. Em 2005, as Ciências do Meio Ambiente, compreendendo as áreas Ecologia e Poluição Ambiental, não são mais representadas como grande área. A Ecologia aparece como área das Ciências Biológicas.

As mudanças de agrupamento das áreas do conhecimento em grandes áreas da SBPC no tempo sofreram mudanças na seqüência de ordenação, nas denominações e no número de grandes áreas - com o mínimo de três e o máximo de oito - como pode ser observado no

QUADRO 2.

Quadro 2 SBPC - Grandes Áreas no Tempo

\begin{tabular}{|l|l|}
\hline \multicolumn{1}{|c|}{1976} & \multicolumn{1}{c|}{1977 -1994 } \\
\hline Seção 1 Ciências Matemáticas & Seção A Ciêncais Aplicadas \\
\hline Seção 2 Ciências da Matéria & Seção B Ciências do Homem \\
\hline Seção 3 Ciências da Terra e do Universo & Seção C Ciêncais Matemáticas \\
\hline Seção 4 Ciências da Vida & Seção D Ciências da Matéria \\
\hline Seção 5 Ciências do Homem & Seção E Ciências do Meio Ambiente \\
\hline Seção 6 Ciências Aplicadas & Seção F Ciências da Terra e do Universo \\
\hline Seção 7 Ciências do Meio Ambiente & Seção G Ciências da Vida \\
\hline \multicolumn{1}{|c|}{$\mathbf{1 9 9 5}$ - 2000 } & \\
\hline Seção A Ciências Aplicadas & Seção A Ciências Humanas \\
\hline Seção B Ciências do Homem & Seção B Ciências Biológicas e da Saúde \\
\hline Seção C Ciências Exatas e da Terra & Seção C Ciências Exatas e Tecnológicas \\
\hline Seção D Ciências da Vida & \\
\hline \multicolumn{1}{|c|}{$\mathbf{2 0 0 3 ~ - 2 0 0 5}$} & \\
\hline & \\
\hline A - Ciências Exatas e da Terra & \\
\hline B - Engenharias & \\
\hline C - Ciências Biológicas & \\
\hline D - Ciências da Saúde & \\
\hline E - Ciências Agrárias & \\
\hline F - Ciências Sociais Aplicadas & \\
\hline G - Ciências Humanas & \\
\hline H - Artes, Letras e Lingüística & \\
\hline
\end{tabular}

Em 2005, as oito grandes áreas da SBPC correspondem exatamente às oito grandes áreas do tabela das áreas do conhecimento em vigor do CNPq. Esta constatação evidencia que o número de grandes áreas da tabela do $\mathrm{CNPq}$ parece ser ainda aceito como adequado. No entanto, as diferenças de denominação das grandes áreas no tempo evidenciam que ainda não existe um consenso terminológico, indicando a necessidade de um questionamento contemporâneo neste sentido.

\section{c) A Australian Standard Research Classification (ASRC)}

Um outro exemplo que merece ser observado é a classificação por campos de pesquisa - uma das três classificações para projetos de pesquisa e desenvolvimento - elaborada pelo 
Australian Bureau of Statistics. As outras duas são: classificação por tipo de pesquisa e classificação por objetivos sócio-econômicos.

A versão de 1993 dessa classificação de campos de pesquisa compreendia 12 grandes áreas. Na versão de 1998, a ASRC amplia seu escopo de interesse para os cursos de ensino superior, ampliando de 12 para 24 o número de classes principais, como pode ser observado no QUADRO 3.

Quadro 3 Comparação ASRC/RFCD 1998 - ASRC/FOR 1993

Australian Standard Research Classification (ASRC/RFCD) 1998
Australian Standard Research Classification (ASRC/FOR) 1993

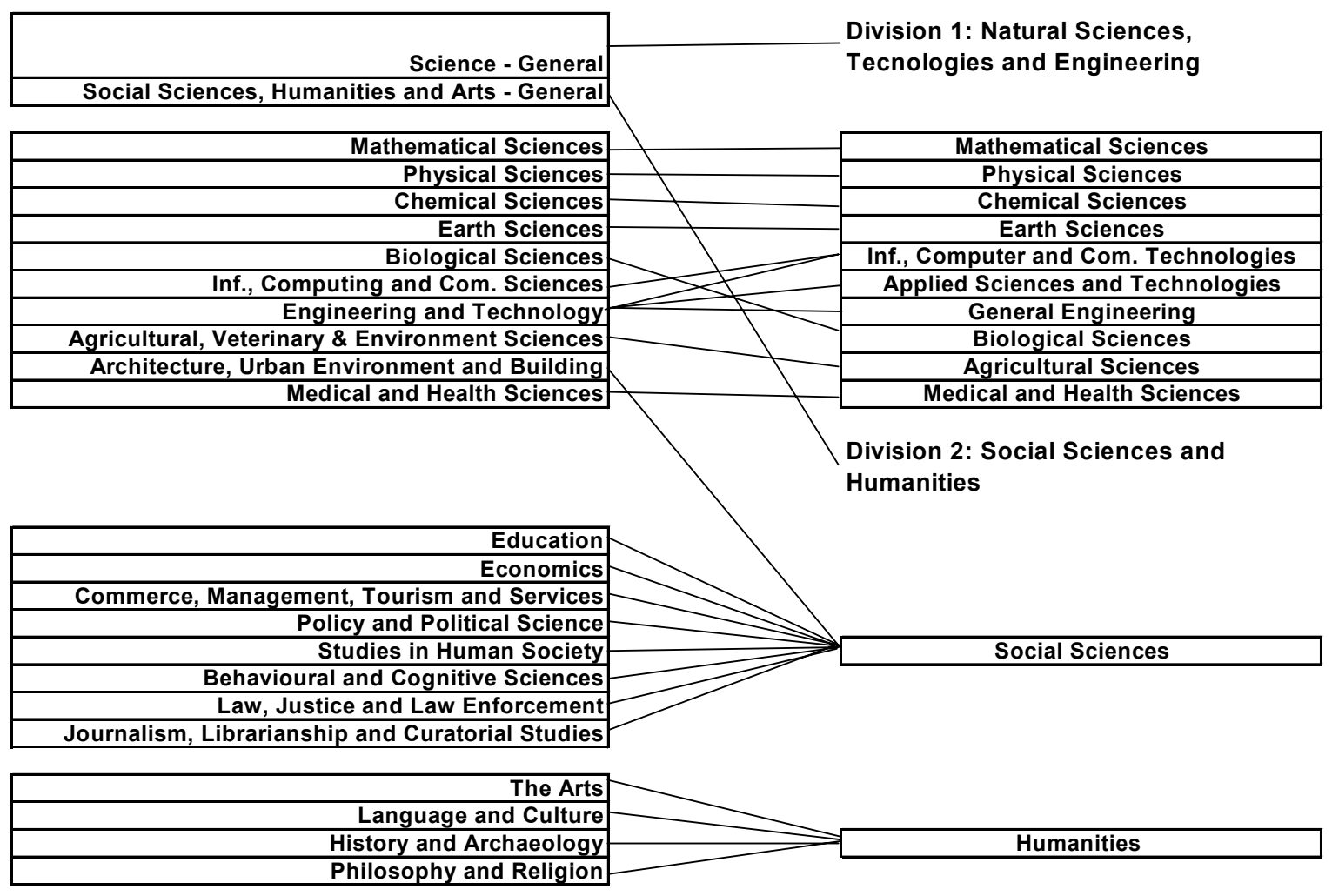

As grandes áreas que se mantiveram as mesmas nas duas versões foram: Ciências Matemáticas; Ciências Físicas; Ciências Químicas; Ciências da Terra; Ciências Biológicas; Ciências Agrárias; Ciências Médicas e da Saúde; Ciências da Informação, Computação e Comunicação; Engenharia e Tecnologia.

A principal modificação da versão de 1993 para a versão de 1998 foi o aumento de classes principais das áreas das Ciências Sociais de uma grande área para oito e, das Humanidades de uma para quatro, equilibrando o número de grandes áreas da Ciência com o número das Ciências Sociais, Humanidades e Artes. Este é um equilíbrio desejável na representação das grandes áreas do universo de $\mathrm{C} \& \mathrm{~T}$, demonstrando o espaço conquistado no 
tempo pelo desenvolvimento de pesquisa e ensino no escopo das ciências humanas, ciências sociais e humanidades.

No caso da classificação australiana (ASRC) é importante destacar que a finalidade da classificação, ou seja, a coleta de dados estatísticos sobre os cursos de educação superior e unidades de atividades de ensino, é diferente das finalidades típicas de uma agência de financiamento de C,T\&I. Portanto, é um exemplo importante de se observar enquanto estratégia de abordagem da produção do conhecimento no contexto do ensino e da pesquisa.

\section{d) Abordagem temática dos saberes no contexto da Educação.}

Em 1998, Edgar Morin, apresenta ao então ministro da Educação da França, desafios visando a desencadear um processo de reforma, na reeducação dos educadores. Esses desafios foram apresentados sob a forma de oito jornadas temáticas que expressam uma maneira de olhar os saberes e, por esta razão, interessam ao presente estudo. A seguir, a ordem temática das jornadas demonstrando, nas próprias palavras de Morin, o "movimento por meio do caminhar" da reforma do ensino: o Mundo, a Terra, a Vida, a Humanidade, Línguas, Civilizações, Literatura, Artes, Cinema, a História, as Culturas Adolescentes, a Religação dos Saberes.

Em sua lógica do caminhar, Morin sistematiza e ordena da seguinte forma: Do Mundo Físico, ressalta o Cosmos e a Terra representado pelas Ciências Exatas e o Conhecimento do Mundo. Do Mundo Biológico, ressalta a Vida representada pelas Ciências Biológicas, situando o Homem na evolução da vida. As Humanidades, representadas pelas Ciências Humanas, levando o Homem a discernir sobre seu destino individual, social, histórico, econômico, imagético, místico e religioso. (Morin, 2001)

\section{e) A Tabela de Áreas do Conhecimento do CNPq}

A presente versão da tabela de classificação das áreas do conhecimento em vigor no CNPq e na CAPES foi estabelecida em 1986. É de natureza hierárquica em quatro níveis: grande área, área, sub-área e especialidade. Apresenta 8 grandes áreas assim nomeadas e nesta ordem:

1. Ciências Exatas e da Terra

2. Ciências Biológicas

3. Engenharias

4. Ciências da Saúde

5. Ciências Agrárias

6. Ciências Sociais Aplicadas

7. Ciências Humanas 


\section{Lingüística, Letras e Artes}

$\mathrm{Na}$ presente década, naturalmente por necessidade de incorporação de novas especificações de áreas, sub-áreas e especialidades, fruto do natural desenvolvimento de $\mathrm{C} \& \mathrm{~T}$, assim como para atender a necessidades específicas, tanto no CNPq quanto na CAPES, a tabela foi acrescida de uma nova grande área, arrolando itens que não estavam representados na tabela original.

O CNPq abre a Grande Área 9 Outros, arrolando 23 itens numa mesma ordem alfabética representando tanto grandes áreas (Ciências e Ciências Sociais), como áreas ( ex.: Engenharia Têxtil, Engenharia Cartográfica e Engenharia Mecatrônica), sub-áreas (Química Industrial) e especialidades (Administração Hospitalar). Inclui também carreiras, profissões e cursos (Carreira Militar, Carreira Religiosa, Diplomacia e Secretariado).

A CAPES abre a Grande Área 9 Outras, com duas subdivisões: Ensino e Multidisciplinar. A área Multidisciplinar é a área que mais cresce atualmente na abertura de novos cursos de mestrado e doutorado reconhecidos pela CAPES. Em fevereiro de 2005, o número de cursos classificados nessa modalidade era de 112. Numa macro-classificação de assuntos feita pela Comissão de Cursos Multidisciplinares, foram identificados quatro agrupamentos temáticos destes cursos para fins de avaliação em: Meio Ambiente e Agronegócios; Engenharias e Tecnologias; Biomédicas e Saúde; Ciências Humanas e Gestão.

Nenhuma documentação foi encontrada como justificativa para a lógica da ordem seqüencial das grandes áreas da tabela em uso pelo CNPq e pela CAPES. No entanto, podemos dizer que a seqüência se inicia com as ciências formais, de cunho mais teórico (Ciências Exatas e da Terra e Ciências Biológicas), prossegue na direção das aplicações especializadas do conhecimento científico e tecnológico (Engenharias, Ciências da Saúde e Ciências Agrárias) e termina pelo conjunto das ciências humanas, sociais e humanidades (Ciências Humanas e Ciências Sociais Aplicadas, Lingüística, Letras e Artes).

\section{CONSIDERAÇÕES FINAIS}

Como primeira consideração destacamos a principal característica de qualquer classificação: toda classificação está relacionada a um propósito. Os exemplos de estruturas de classificação analisados apresentaram diferentes abordagens de agregação de áreas do conhecimento em grandes áreas, características da época, dos contextos institucionais 
para os quais foram gerados e da cultura dos classificacionistas. As particularidades de cada classificação analisada visaram atender a propósitos previamente definidos que, pela sua natureza, diferenciaram as formas de agregações de áreas do conhecimento em grandes áreas.

Uma segunda consideração diz respeito à caracterização do objeto informação nos diferentes contextos analisados. As classificações bibliográficas, a classificação da SBPC e a classificação australiana lidam essencialmente com a literatura de $C \& T$. As jornadas temáticas de Morin evidenciam as principais temáticas do ensino para profissionais da educação. A tabela de áreas do conhecimento do CNPq-CAPES, apresenta objetivo singular de sistematização de informação sobre $C \& T$, caracterizada como meta-informação para fins de gestão e avaliação.

Uma terceira consideração está na reflexão sobre diferentes necessidades de classificação evidenciando a diversidade de pontos de vista dos objetos de estudo com a finalidade da representação. A classificação da ciência, fruto do trabalho do cientista, tem por finalidade a classificação dos objetos investigados, por exemplo: plantas, animais, numa clara hierarquia de gênero-espécie, visando a completude máxima das classes. A classificação da produção científica publicada tem por objetivo sistematizar, para fins de acesso e uso, a literatura produzida pelas diversas áreas do conhecimento, ordenadas numa hierarquia de diferentes níveis de agregação: super-ordenação em grandes áreas e sub-ordenação em subáreas e especialidades, evidenciando uma estrutura integrativa todo-parte.

No caso dos esquemas de classificação bibliográfica e tabelas de classificação que ordenam a literatura, a definição das grandes áreas foi fortemente influenciada por abordagem filosófica. No entanto, o número e a denominação de grandes áreas apresentaram diferenças. Algumas questões relacionadas à agregação de áreas do conhecimento em grandes áreas puderam ser identificadas como importantes pontos de reflexão para uma tabela de classificação com finalidade de gestão e avaliação.

A composição de áreas de conhecimento em grandes áreas deve ser sempre consistente respeitando o princípio de divisão adotado. No entanto, dependendo das necessidades específicas de agregação, o número de grandes áreas pode variar, assim como as suas denominações e a seqüência de ordenação.

A construção de uma tabela de classificação das áreas do conhecimento para uso das agências de fomento se revela de natureza complexa. A primeira dificuldade vem da caracterização dos diferentes pontos de vista dos principais usuários frente a um mesmo instrumento: por um lado, os gestores, administradores e técnicos em suas necessidades operacionais dos programas das agências; por outro lado, a comunidade científica na busca de 
encontrar uma forma de classificar seus interesses específicos de pesquisa para demandas de fomento.

Do ponto de vista do cientista, a maior preocupação está vinculada a questões da coerência da co-extensividade do todo com as partes na representação dos níveis hierárquicos da tabela, sendo que o nível de maior interesse da comunidade é, sem dúvida, o da especialidade. Do ponto de vista das agências de fomento a representação das grandes áreas numa tabela de classificação de áreas do conhecimento é de natureza político-institucional em função de objetivos de uso gerencial. Neste caso especial, a definição de um número restrito de grandes áreas dificulta a sistematização adequada para fins de gestão e avaliação e para a geração de séries históricas. Portanto, as necessidades de uso e as expectativas de representação são diferentes, mas precisam ser harmonizadas face ao mesmo instrumento de classificação, o que evidencia a complexidade da construção de tal instrumento.

A definição do conceito de grande área, área, subáreas e especialidade do conhecimento e a definição do princípio de divisão a ser adotado devem ser claramente especificados e aplicados de forma coerente. Esses serão os critérios norteadores da estrutura e da composição dos elementos em tabela de classificação de projetos de pesquisa e desenvolvimento para uso de agências de fomento e na produção de séries estatísticas nacionais.

O número de grandes áreas deve ser suficientemente adequado para permitir sistematizações temáticas consistentes. As denominações terminológicas devem estar conforme a nomenclatura corrente. As grandes áreas devem apresentar coerência em relação ao conjunto de áreas do conhecimento que abrigam.

A questão da inter-multidisciplinaridade sempre e cada vez mais presente como característica da pesquisa e do ensino em C\&T não foi devidamente contemplada em nenhuma das classificações analisadas. Os exemplos apresentados foram definidos em base de uma estrutura hierárquica estabelecida por padrões canônicos de disciplinaridade. A vasta literatura sobre este assunto ainda não contempla procedimentos relacionados à organização e representação do conhecimento de natureza multidisciplinar em tabelas e esquemas de classificação em ciência e tecnologia.

Para concluir, ficamos com as palavras de Morin mostrando a singularidade, a complexidade e a necessidade da organização do conhecimento:

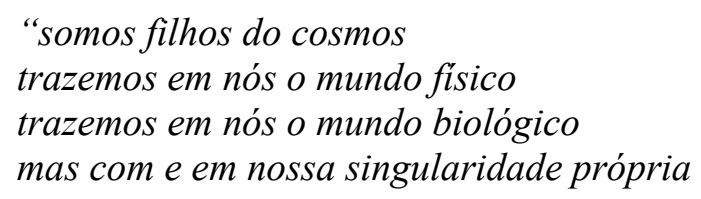


em outras palavras: para enfrentarmos o desafio da complexidade, precisamos de princípios organizadores do conhecimento (grifo nosso)

Morin evidencia de maneira ainda mais clara o importante papel da organização do conhecimento em diferentes contextos de informação, deixando uma mensagem que pode ser interpretada como direcionada aos pesquisadores da área da Ciência da Informação, evidenciando que a organização do conhecimento é vista como uma arte, com funções claras, relevantes e específicas de natureza científica:

e, mais ainda:

$$
\begin{aligned}
& \text { "... arte de organizar o pensamento, religar e } \\
& \text { diferenciar, contextualizar e globalizar" }
\end{aligned}
$$

"informação e conhecimento relacionados a contexto e conjunto",

todas estas, características chave do tema central deste trabalho que é a discussão em torno da agregação das áreas do conhecimento em contextos previamente definidos.

\section{REFERÊNCIAS}

ANDERSEN, J. Communication technologies and the concept of knowledge organization - a medium-theory perspective. Knowledge Organization, v. 29, n. 1, 2002. p. 29-39 AUSTRALIAN BUREAU OF STATISTICS. Australian Standard Research Classification 1998: a set of classifications for R\&D projects. T.J. Skinner, acting Australian Statistician. Canberra: ABS, 1998

AUSTRALIAN BUREAU OF STATISTICS. Australian Standard Research Classification 1993: a set of classifications for R\&D projects. Ian Castles, Australian Statistician. Canberra: ABS, 1993.

BARBOSA, Alice P. Teoria e prática dos sistemas de classificação bibliográfica. Rio de Janeiro: IBBD, 1969.

BLISS, H. E. The organization of knowledge in libraries. New York: H.W. Wilson, 1939 CAPES. Comissão dos cursos multidisciplinares. Documento de área triênio 1998-2000; triênio 2001-2003. Disponível em: http://www.capes.gov.br/capes/portal/, acesso em $15 / 05 / 2005$.

DAHLBERG, I. Knowledge organization and terminology: philosophical and linguistic bases. International Classification. v.19, n.2, p.65-71, 1992.

DAHLBERG, I. Ontical structures and universal classification. Bangalore: Sarada Ranganthan Endowment,1978.

FOSKETT, D.J. Classification and indexing in the social sciences. London: Butterworths, 1963.

KAULA, P. N. Repensando os conceitos no estudo da Classificação. (Baseado no artigo submetido à 4 Conferência sobre Pesquisa em Classificação, Augsburg Alemanha Ocidental, de 20 de junho a 2 de julho de 1982) http://www.conexaorio.com/biti/Kaula/index.htm).

KNOWLEDGE ORGANIZATION: concept theory, classification, indexing and knowledge representation. v. 28, no. 2, 2001 
KUMAR, K. (1981) Theory of classification. 2.ed. New Delhi: Vikas Publishing House, 1981. 538p.

LANGRIDGE, D. Classification and indexing in the humanities. London: Butterworths, 1976.

LANGRIDGE, D. W. Classificação: abordagem para estudantes de biblioteconomia.

Tradução de Rosali P. Fernandez. Rio de Janeiro, Interciência, 1977.

MILLS, J. A . A modern outline to library classification. London: Chapman \& Hall, 1960.

MORIN, E. Introdução ao pensamento complexo. 2. ed. Lisboa: Instituto Piaget, 1990.

MORIN, E. A cabeça bem-feita: repensar a reforma, reformar o pensamento. Rio de Janeiro:

Bertrand Brasil, 2001.

MORIN, E. A religação dos saberes: o desafio do século XXI. . Rio de Janeiro: Bertrand Brasil, 2001.

NEEDHAM, C. D. Organizing knowledge in libraries. London: A . Deutsch, 1964

OLSON, H. A . Diversity in knowledge organization. Editorial. Knowledge Organization, v. 28, no. 2, p.63-64, 2001.

PALMER, B.I.. Itself an education: lectures on classification. London: Library Association, 1971.

RANGANATHAN, S.R. Philosophy of library classification. New Delhi: Ejnar

Munksgaard, 1951.

RANGANTHAN, S.R. Prolegomena to library classification. Bombay: Asia Publishing House, 1967.

SAYERS, W. C. A manual of classification for librarians and bibliographers. London:

Grafton, 1955

SOUZA, Rosali Fernandez. Áreas do Conhecimento. DataGramaZero, v. 5, n. 2,

Abr. 2004. Disponível em http://www.datagramazero.org.br/ago04/F I onum.htm

VICKERY, B. C. Classificação e indexação nas ciências. Rio de Janeiro: BNG/Brasilart, 1980. 274p.

VICKERY, B. C. Knowledge Representation: a brief review. Journal of Documentation, v. 42, no 3, sept. 1986. p. 145-159.

\begin{abstract}
Knowledge organization is a key area of research and teaching in Information Science. Published literature in this field mainly deals with the process, activities and instruments developed for the treatment of documents regarding storage, dissemination, retrieval and use in information systems. Different types and needs of information have demonstrated the diversity in knowledge organization in other application contexts. The present work has explanatory purpose. It analyses examples of tables and schemes of classification in science and technology aiming to identify aggregations of areas of knowledge at super-ordination level for different needs of information production and use. The instruments analysed were: bibliographical classification schemes, areas of knowledge classification tables for purposes of communication in science, science administration and production of statistics in science. It also presents a thematic approach of science in the context of education. Results showed that exists a consensus of areas of knowledge aggregation at super-ordination level, although the number and order of presentation may vary regarding the nature of the object to be represented, as well as the specific purpose of knowledge organization.
\end{abstract}

KEYWORDS: Knowledge Organization. Information Representation. Classifications in Science and Technology. Areas of Knowledge.

Originais recebidos em 13/12/ 2005. 